\title{
Identification of Linear Time Invariant Systems Using FMCW Signals and Stretch Processing Receivers
}

\author{
Stephan Häfner and Reiner Thomä
}

\begin{abstract}
The paper deals with the identification of linear time invariant (LTI) systems by a special observer. An observer emitting an frequency modulated continuous wave (FMCW) signal and having a stretch processor as receiver will be considered for system identification. A thorough derivation of the gathered baseband signal for arbitrary LTI systems will be given. It is shown, that the received signal is approximately given by the transfer function of the LTI system over the frequency sweep of the FMCW signal. The proof relies on an infinite large time-bandwidth product of the transmit signal, such that errors remain in practical applications with a finite time-bandwidth product. Monte-Carlo simulations are conducted to verify the approximation and to quantify its accuracy and remaining errors. The findings are important for e.g. calibration or derivation of a device model in FMCW radar applications.
\end{abstract}

Keywords-FMCW signal, LTI system, stretch processing receiver, system identification.

\section{INTRODUCTION}

System identification is the task of inferring a model of a physical system from observed input-output data [1], [2], [3]. This task occurs in many areas of engineering, as e.g. in radar [4], [5] and channel sounding [6], [7], [8], and also in control engineering or characterisation of hardware components like filters, transmission lines, antennas etc. In many applications, the physical system under identification is modelled as linear and time invariant (LTI system). The system identification is accomplished by an observer, which excites the physical system with a known signal (input) and gathers the response (output). Real-time sampling of the response signal at baseband requires analog-to-digital converters (ADCs) with sampling rates of at least twice the signal bandwidth. In case of a large signal bandwidth (e.g. several GHz) appropriate digitisers are costly. However, if the system under identification is time-invariant or slowly time-varying, real-time acquisition is not necessary. Periodic sub-sampling can be employed in that case, such that the digitiser requirements are relaxed to the cost of a longer measurement duration [4], [9]. In this paper, an observer which emits an frequency modulated continuous wave (FMCW) signal and having a stretch processing receiver architecture is considered as observer [10], [11]. Stretch

Manuscript received March 10, 2019; revised July 3, 2019.

Stephan Häfner and Reiner Thomä are with the Electronic Measurements and Signal Processing Group at Technische Universität Ilmenau, Ilmenau, 98684 Germany (e-mail: (stephan.haefner,reiner.thomae)@tu-ilmenau.de).

Stephan Häfner is funded by the Deutsche Forschungsgemeinschaft under Grant no. 317632307.

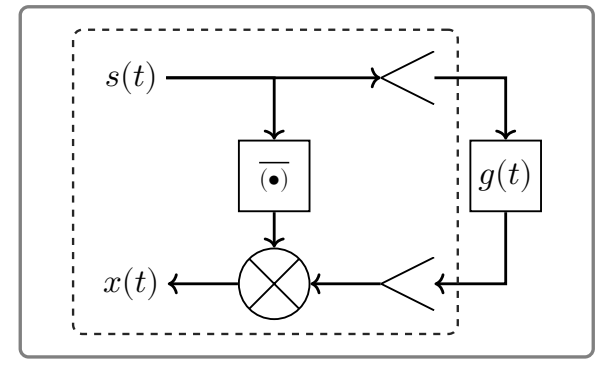

Fig. 1. Block diagram of the considered system identification setup, featuring the observer with a stretch processing receiver structure (dashed box), and the LTI system under identification $g(t)$. Symbol $\overline{(\bullet)}$ denotes the complex conjugate operator.

processing receivers are matched-filter realisations for FMCW signals with a large time-bandwidth product [12]. Advantage of such a receiver is, that it can be realised with a moderate hardware effort and ADCs with a low sampling rate can be employed. Figure 1 shows a block diagram of the considered observer. For a delay and damping LTI system, the baseband signal $x(t)$ is known from literature [12], [13]. However, to the best of the authors knowledge, a thorough derivation of the baseband signal for an arbitrary LTI system is not given in literature. Knowledge of the baseband signal for arbitrary LTI systems is important to derive a device model of the observer for high-resolution parameter estimation [5] or to calibrate the observer itself [14]. In this paper, the baseband signal will be analytically derived under several assumptions, and the respective limitations and occurring errors will be elaborated. The finding has been recently used by the authors to develop a device model for an FMCW radar, see [5], whereas no proof has been given.

Under narrowband assumption, an FMCW signal in the complex passband domain can be represented as [12]

$$
s(t)=a \cdot \exp \left\{\jmath\left(\pi \frac{B}{T} t^{2}+2 \pi f_{c} t\right)\right\} \quad, \quad|t| \leq \frac{T}{2}
$$

Variables $T, B$ and $f_{c}$ denote the sweep time, swept bandwidth and the centre frequency, respectively. The signal is transmitted over a LTI system, which is described by its respective time $g(t)$ and frequency $G(f)$ domain representation. In Section II it will be shown, that the output of the LTI system for large 
time-bandwidth products $(T \cdot B>>1)$ is

$$
s(t) * g(t) \approx s(t) \cdot G\left(\frac{B}{T} t+f_{c}\right) .
$$

The output signal $x(t)$ of the stretch processing receiver is given by

$$
x(t)=\overline{s(t)} \cdot[s(t) * g(t)],
$$

whereas $\overline{(\bullet)}$ denotes the complex conjugate operator. Plugging (2) in (3) yields an approximation of the baseband signal

$$
x(t) \approx \overline{s(t)} \cdot s(t) \cdot G\left(\frac{B}{T} t+f_{c}\right)=a^{2} \cdot G\left(\frac{B}{T} t+f_{c}\right) .
$$

Thus, the baseband signal in time domain is proportional to the transfer function of the LTI system over the frequency sweep of the FMCW signal. Because the time-bandwidth product cannot be infinite in practise, some errors remain and the relation is only approximately true. In Section III the accuracy of the approximation and the introduced errors will be addressed by simulations.

\section{A. Limitations}

Application of a stretch processing receiver has some limitations and constraints regarding the LTI system under identification.

The transmit signal undergoes a delay while being transmitted over the LTI system. A delayed receive signal causes a loss in resolution due to the corresponding loss in bandwidth, as shown in [15]. The resolution is not degraded, if the delay is much less than the sweep time. Restate the transfer function of the LTI system in terms of magnitude and phase

$$
G(f)=|G(f)| \cdot \exp \{\jmath \varphi(f)\} \quad .
$$

If the magnitude of the group delay of the LTI system fulfils

$$
\left|-\frac{1}{2 \pi} \frac{\partial \varphi(f)}{\partial f}\right|<<T,
$$

the resolution is not degraded due to the transmission delay. Hence, a large sweep time or equivalently a large measurement duration is required, which is a drawback of this kind of observer systems.

In practical implementations of the stretch processing receiver, a low-pass filter is present at the output of the multiplier of the stretch processor [5]. This low-pass filter has no influence on approximation (4) and can be neglected in the mathematical derivations, if the impulse response duration $\sigma_{\text {LTI }}$ of the LTI system is related to the cut-off frequency $f_{\text {cut-off }}$ of the low-pass filter by

$$
\sigma_{\text {LTI }} \leq \frac{T}{B} f_{\text {cut-off }} .
$$

Considering the ADC sampling rate $f_{\mathrm{s}}$, which has to fulfil $f_{\mathrm{s}} \geq 2 f_{\text {cut-off }}$ according to Nyquist's sampling theorem, the maximum measurable impulse response duration is

$$
\sigma_{\mathrm{LTI}} \leq \frac{T}{B} \frac{f_{\mathrm{s}}}{2}
$$

\section{Proof of Approximation (2)}

The output of a LTI system excited with the FMCW signal in (1) is given by

$$
s(t) * g(t)=\int_{-\infty}^{\infty} S(f) \cdot G(f) \cdot \exp \{\jmath 2 \pi t f\} \mathrm{d} f \quad .
$$

The spectrum $S(f)$ of an FMCW signal is [12]

$$
S(f)=\sqrt{\frac{a^{2} T}{2 B}} \exp \left\{-\jmath \pi \frac{T}{B}\left(f-f_{c}\right)^{2}\right\}\left[Z\left(q_{1}\right)+Z\left(q_{2}\right)\right]
$$

with

$$
\begin{aligned}
q_{1} & =\sqrt{\frac{T}{2 B}}\left[B+2\left(f-f_{c}\right)\right] \\
q_{2} & =\sqrt{\frac{T}{2 B}}\left[B-2\left(f-f_{c}\right)\right] .
\end{aligned}
$$

Function $Z(q)$ denotes the complex Fresnel integral [16]

$$
Z(q)=\int_{0}^{q} \exp \left\{\jmath \frac{\pi}{2} x^{2}\right\} \mathrm{d} x .
$$

For large time-bandwidth products, the values of $q_{1}$ and $q_{2}$ tend to infinity, and magnitude and phase of $Z\left(q_{1}\right)+Z\left(q_{2}\right)$ in the frequency range $\left[f_{c}-B / 2, f_{c}+B / 2\right]$ can be approximated as [12]

$$
\begin{aligned}
\lim _{T \cdot B \rightarrow \infty}\left|Z\left(q_{1}\right)+Z\left(q_{2}\right)\right| & =\sqrt{2} \\
\lim _{T \cdot B \rightarrow \infty} \arg \left\{Z\left(q_{1}\right)+Z\left(q_{2}\right)\right\} & =\frac{\pi}{4}
\end{aligned} .
$$

Figure 2 shows magnitude and phase of the Fresnel integral sum $Z\left(q_{1}\right)+Z\left(q_{2}\right)$ for different sweep times and bandwidths over the frequency range from $-6 \mathrm{GHz}$ to $6 \mathrm{GHz}$ and a centre frequency of $0 \mathrm{~Hz}$. Ripples at the band edges (denoted as Fresnel oscillations) are present, which rise due to the waveform uncertainty principle (Gabor's uncertainty principle) [12]. Hence, the Fresnel oscillations reduce, if the sweep time increases. An increase of the bandwidth does not reduce the Fresnel oscillations at the band edges, but the oscillations in the band middle decrease. Because the approximations are only valid on the interval $\left[f_{c}-B / 2, f_{c}+B / 2\right]$ and the spectrum magnitude rapidly decreases outside of this interval, the magnitude can be approximated by a rectangular function. Plugging the approximations into 10 yields the signal spectrum for large time-bandwidth products

$S(f) \approx \sqrt{\frac{a^{2} T}{B}} \exp \left\{-\jmath \pi \frac{T}{B}\left(f-f_{c}\right)^{2}+\jmath \frac{\pi}{4}\right\} \operatorname{rect}\left(\frac{f-f_{c}}{B}\right)$.

Plugging the approximative signal spectrum into 9 yields

$$
\begin{aligned}
& s(t) * g(t) \approx \sqrt{\frac{a^{2} T}{B}} \cdot \exp \left\{\jmath \frac{\pi}{4}\right\} \\
& \cdot \int_{f_{c}-B / 2}^{f_{c}+B / 2} G(f) \exp \left\{-\jmath \pi \frac{T}{B}\left(f-f_{c}\right)^{2}\right\} \exp \{\jmath 2 \pi t f\} \mathrm{d} f .
\end{aligned}
$$

Thus, the convolution result is given by the integral of an oscillating function with envelope $G(f)$. 

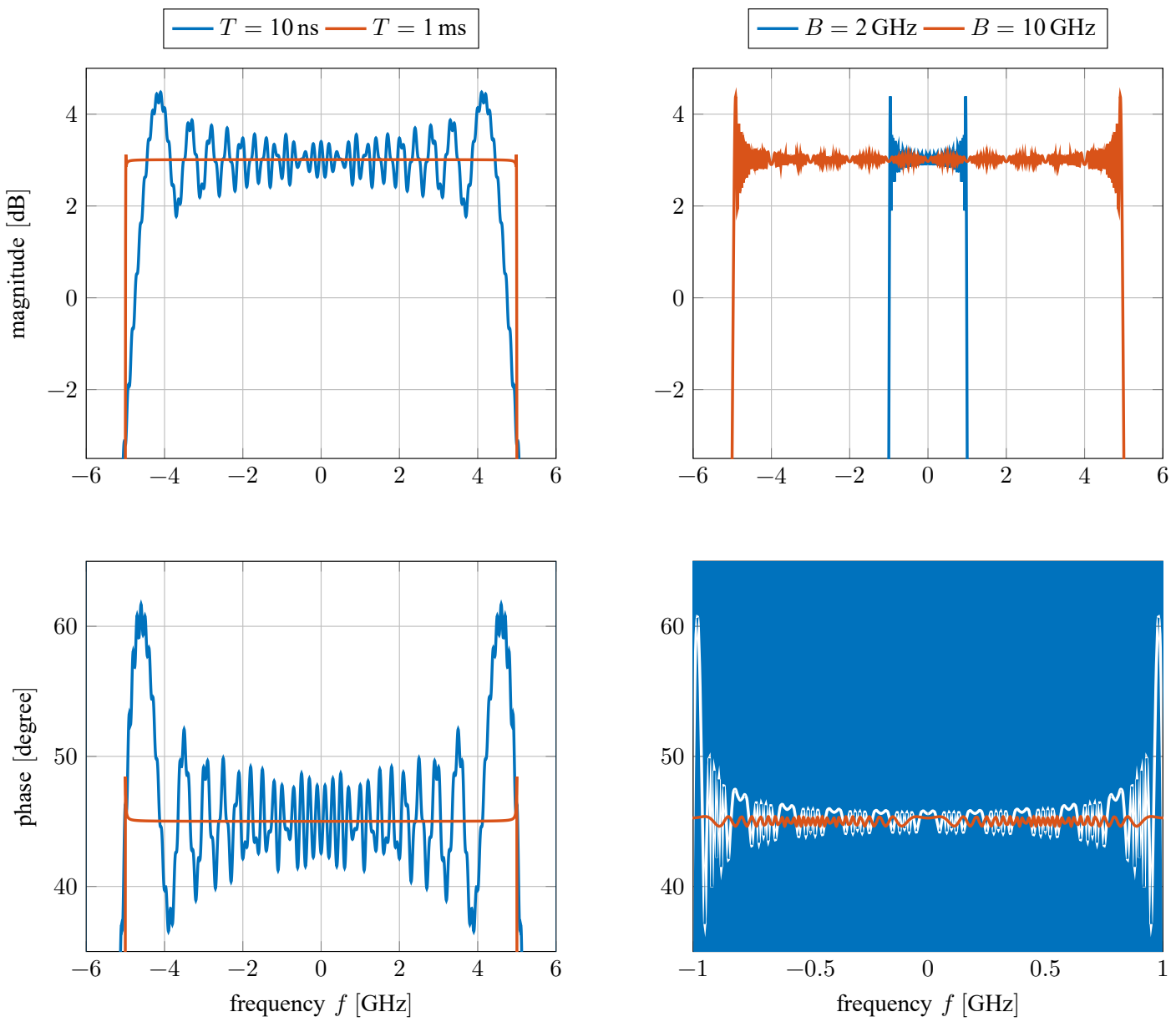

Fig. 2. Magnitude and phase of the complex Fresnel integral sum $Z\left(q_{1}\right)+Z\left(q_{2}\right)$ for various sweep times and bandwidths. For variable sweep time, the swept bandwidth was $10 \mathrm{GHz}$; and for variable swept bandwidth the sweep time was $1 \mu \mathrm{s}$.

The principle of stationary phase [12] will be applied to approximate the integral in (15). Assuming the oscillating function as rapidly varying and the envelope as sufficiently smooth, contributions of the integrand cancel approximately out under the integral. Hence, dominant contributions occur only in the regions of minimal oscillation (stationary points), and at the end points of the integration interval. Under the first order approximation, the contributions of the end points become negligible in comparison to contribution of the stationary points [17]. For sake of convenience, the phase term of the integrand is rewritten as

$$
\Psi(f)=2 \pi f\left(t+\frac{T}{B} f_{c}\right)-\pi \frac{T}{B} f^{2}-\pi \frac{T}{B} f_{c}^{2} .
$$

The second order Taylor series expansion of the phase term, evaluated at point $f_{0}$, gives

$$
\Psi(f) \approx \Psi\left(f_{0}\right)+\dot{\Psi}\left(f_{0}\right)\left(f-f_{0}\right)+\frac{1}{2} \ddot{\Psi}\left(f_{0}\right)\left(f-f_{0}\right)^{2},
$$

with

$$
\begin{aligned}
& \dot{\Psi}(f)=\frac{\mathrm{d} \Psi(f)}{\mathrm{d} f}=2 \pi\left(t+\frac{T}{B} f_{c}\right)-2 \pi \frac{T}{B} f \\
& \ddot{\Psi}(f)=\frac{\mathrm{d}^{2} \Psi(f)}{\mathrm{d} f^{2}}=-2 \pi \frac{T}{B} .
\end{aligned}
$$

According to the stationary phase principle, the evaluation point $f_{0}$ is selected to be the point of vanishing first order derivative $(\dot{\Psi}(f)=0)$, because the oscillations are minimal their. The only stationary point is at $f_{0}=\frac{B}{T} t+f_{c}$. Plugging the Taylor series expansion into 15 and noting that the integral is only significant in the $\epsilon$ vicinity of the stationary point, yields

$$
\begin{aligned}
s(t) * g(t) \approx & \sqrt{\frac{a^{2} T}{B}} \exp \left\{\jmath \frac{\pi}{4}\right\} \exp \left\{\jmath \Psi\left(f_{0}\right)\right\} \\
& \cdot \int_{f_{0}-\epsilon}^{f_{0}+\epsilon} G(f) \exp \left\{\jmath \frac{1}{2} \ddot{\Psi}\left(f_{0}\right)\left(f-f_{0}\right)^{2}\right\} \mathrm{d} f .
\end{aligned}
$$

Assuming the envelope as slowly varying and $\ddot{\Psi}\left(f_{0}\right)\left(f-f_{0}\right)^{2}$ as sufficiently large, $G(f)$ contributes to the integral only in the vicinity of the stationary point $f_{0}$. Hence, $G(f)$ can be approximated by $G\left(f_{0}\right)$, which yields

$$
\begin{aligned}
s(t) * g(t) \approx & \sqrt{\frac{a^{2} T}{B}} \exp \left\{\jmath \frac{\pi}{4}\right\} \exp \left\{\jmath \Psi\left(f_{0}\right)\right\} G\left(\frac{B}{T} t+f_{c}\right) \\
& \cdot \int_{f_{0}-\epsilon}^{f_{0}+\epsilon} \exp \left\{\jmath \frac{1}{2} \ddot{\Psi}\left(f_{0}\right)\left(f-f_{0}\right)^{2}\right\} \mathrm{d} f \cdot(20)
\end{aligned}
$$

Since the main contributions to the integral are concentrated around the stationary point, a small error will occur by 
integrating over the infinite domain. Considering furthermore the substitution $\xi^{2}=\frac{1}{2} \ddot{\Psi}\left(f_{0}\right)\left(f-f_{0}\right)^{2}$ and the complex Fresnel integral over the infinite domain [16], the integral in (20) can be approximated as

$$
\begin{aligned}
& \int_{f_{0}-\epsilon}^{f_{0}+\epsilon} \exp \left\{\jmath \frac{1}{2} \ddot{\Psi}\left(f_{0}\right)\left(f-f_{0}\right)^{2}\right\} \mathrm{d} f \\
\approx & \int_{-\infty}^{\infty} \exp \left\{\jmath \frac{1}{2} \ddot{\Psi}\left(f_{0}\right)\left(f-f_{0}\right)^{2}\right\} \mathrm{d} f \\
= & \sqrt{\frac{2 \pi}{\ddot{\Psi}\left(f_{0}\right)}} \cdot \int_{\xi(f=-\infty)}^{\xi(f=\infty)} \exp \left\{\jmath \xi^{2}\right\} \mathrm{d} \xi \\
= & \sqrt{\frac{2 \pi}{\ddot{\Psi}\left(f_{0}\right)}} \cdot \exp \left\{\jmath \frac{\pi}{4}\right\}=\sqrt{\frac{B}{T}} \cdot \exp \left\{-\jmath \frac{\pi}{4}\right\} .
\end{aligned}
$$

Plugging this result into 20 yields the approximative output signal in time domain

$$
\begin{aligned}
s(t) * g(t) \approx & \sqrt{\frac{a^{2} T}{B}} \exp \left\{\jmath \frac{\pi}{4}\right\} \exp \left\{\jmath \Psi\left(f_{0}\right)\right\} G\left(\frac{B}{T} t+f_{c}\right) \\
& \cdot \sqrt{\frac{B}{T}} \exp \left\{-\jmath \frac{\pi}{4}\right\} \\
= & a \cdot \exp \left\{\jmath \Psi\left(f_{0}\right)\right\} \cdot G\left(\frac{B}{T} t+f_{c}\right) \\
= & a \cdot \exp \left\{\jmath\left(\pi \frac{B}{T} t^{2}+2 \pi f_{c} t\right)\right\} \cdot G\left(\frac{B}{T} t+f_{c}\right) \\
= & s(t) \cdot G\left(\frac{B}{T} t+f_{c}\right),
\end{aligned}
$$

which has been stated in (2).

\section{VERIFICATION AND ERROR ANALYSIS}

Basically, the approximations in Section II introduce errors. Under first order approximation, these errors can be assumed as additive. Hence, the output of the stretch processor in the noiseless case can be represented as

$$
x(t)=a^{2} \cdot G\left(\frac{B}{T} t+f_{c}\right)+\varepsilon(t),
$$

with $\varepsilon(t)$ accounting for the approximation errors. According to the assumption of a large time-bandwidth product, the errors should fulfil

$$
\lim _{T \cdot B \rightarrow \infty} \varepsilon(t)=0
$$

Monte-Carlo simulations with varying system settings as e.g. varying sweep time or bandwidth will be carried out to verify (24). Randomly generated LTI systems are considered for the simulation. A LTI system can be represented in frequency domain by its corresponding rational form [3]

$$
G(s=\jmath 2 \pi f)=k \frac{\prod_{r=1}^{Z}\left(s-z_{r}\right)}{\prod_{r=1}^{P}\left(s-p_{r}\right)},
$$

with the zeros $z_{r} \in \mathbb{C}$ and poles $p_{r} \in \mathbb{C}$. For sake of convenience, the gain factor $k$ is set to $k=1$. In each MonteCarlo run, the zeros and poles are uniformly and pairwise complex conjugately generated in the range from $50 \mathrm{kHz}$ to $100 \mathrm{kHz}$. The range is chosen in order to have a low group delay for small numbers of poles and zeros. The amplitude of the transmit signal is chosen as $a=1$ and the number of Monte-Carlo runs is set to $M=1000$. As figure of merit, the normalised root mean-square error (RMSE) is calculated

$$
\sqrt{\frac{1}{N M} \sum_{m=1}^{M} \sum_{n=1}^{N}\left\|x_{m}\left(n t_{0}\right)-G_{m}\left(n \frac{B}{T} t_{0}+f_{c}\right)\right\|_{2}^{2}}
$$

with $G_{m}$ the generated LTI system and $x_{m}$ the calculated baseband signal for the $m$-th Monte-Carlo run. The baseband signal is calculated by considering the system structure given in Fig. 1. The sampling time is $t_{0}=100 \mathrm{~ns}$ and the centre frequency is $f_{c}=0 \mathrm{~Hz}$. Because the number of samples $N$ varies over the sweep time $T$ according to relation $T=N t_{0}$, the RMSE has been normalised.

First, simulations for various sweep times and bandwidths, and a fixed number of poles and zeros $Z=P=4$ are considered. The RMSE curves are shown in Fig. 3, indicating a decreased error if the time or bandwidth increases. Hence, the error decreases with an enlarging time-bandwidth product, which indicates the correctness of (24).

Second, simulations for various sweep times and orders (max. number of poles and zeros) of the LTI system are considered, whereas the swept bandwidth was $1 \mathrm{MHz}$. An increasing system order causes a higher group delay, which decreases the resolution and hence increases the approximation error. Furthermore, a higher order causes a transfer function which is less smooth compared to the oscillations of the FMCW signal. Hence, the stationary phase approximation becomes less accurate and the approximation error increases. The RMSE curves in Fig. 4 verify the stated relation between group delay and approximation error.

Last, simulations with varying fractional bandwidth $B / f_{c}$ are conducted, whereas the centre frequency has been varied and the bandwidth was fixed to $100 \mathrm{kHz}$. The RMSE curves are depicted in Fig. 5 , indicating an decreased error for small fractional bandwidths (small bandwidth and high centre frequency). Because the spectral oscillations of the FMCW signal are higher for large centre frequencies and the stimulated part of the envelope of the LTI system becomes smoother for small bandwidths, the approximations by the stationary phase principle become more accurate for small fractional bandwidths.

Summarised, the simulations verify approximation (4) and limitation (6). Furthermore it was found, that the accuracy of the baseband signal approximation improves, if the fractional signal bandwidth reduces.

\section{CONCLUSION}

Identification of linear time invariant systems by an observer which emits an FMCW signal and has a stretch processing receiver architecture has been considered. It has been theoretically proven under the assumption of an infinite timebandwidth product, that the received baseband signal is approximately proportional to the frequency response of the LTI system. The accuracy of this approximation has been addressed in Monte-Carlo simulations with varying sweep time and bandwidth of the FMCW signal, and varying order 


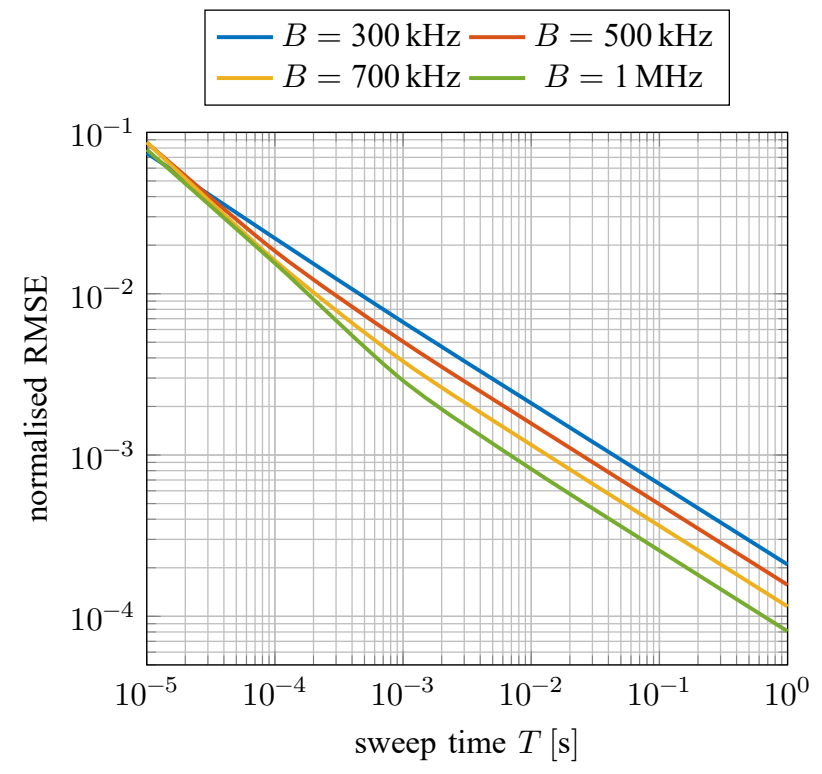

Fig. 3. Normalised RMSE over various sweep times and different bandwidths.

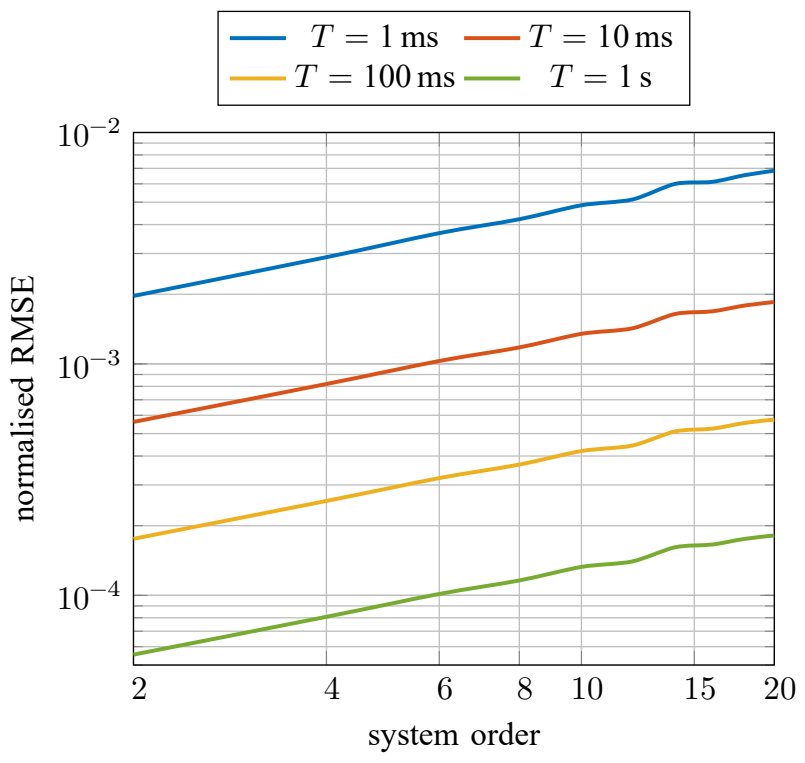

Fig. 4. Normalised RMSE over varying order of the LTI system, and different sweep times. Swept bandwidth was $1 \mathrm{MHz}$

of the LTI system. The simulations verify the approximation and indicate, that the remaining approximation errors decrease if 1) the time-bandwidth product enlarges, 2) the group delay of the LTI system is much smaller than the sweep time or 3 ) the fractional bandwidth is small.

\section{REFERENCES}

[1] L. Ljung, System Identification: Theory for the User, ser. Prentice-Hal Information and System Sciences Series. Prentice-Hall, 1987.

[2] T. Söderström and P. Stoica, Eds., System Identification. Prentice-Hall, Inc., 1989.

[3] R. Pintelon and J. Schoukens, System Identification: A Frequency Domain Approach. IEEE Press, 2001.

[4] R. Zetik, J. Sachs, and R. S. Thomä, "UWB Short-Range Radar Sensing - The Architecture of a Baseband, Pseudo-Noise UWB Radar Sensor," IEEE Instrum. Meas. Mag., vol. 10, no. 2, pp. 39-45, April 2007.

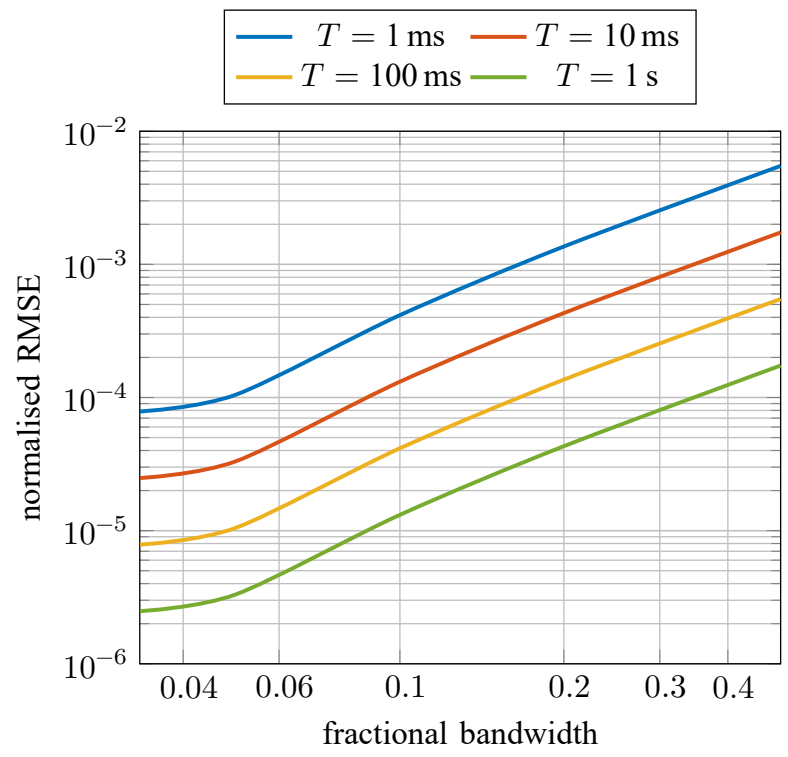

Fig. 5. Normalised RMSE over various fractional bandwidths and different sweep times. Swept bandwidth was $100 \mathrm{kHz}$.

[5] S. Häfner, A. Dürr, R. Thomä, C. Waldschmidt, and G. D. Galdo, "HighResolution Parameter Estimation for Chirp-Sequence Radar Considering Hardware Impairments," in 11th German Microw. Conf. (GeMiC), March 2018, pp. $355-358$.

[6] R. S. Thomä, D. Hampicke, A. Richter, G. Sommerkorn, A. Schneider, U. Trautwein, and W. Wirnitzer, "Identification of Time-Variant Directional Mobile Radio Channels," IEEE Trans. Instrum. Meas., vol. 49 , no. 2, pp. 357-364, April 2000.

[7] R. Müller, S. Häfner, D. Dupleich, R. S. Thomä, G. Steinböck, J. Luo, E. Schulz, Xiaofeng Lu, and Guangjian Wang, "Simultaneous MultiBand Channel Sounding at mm-Wave Frequencies," in 10th Eur. Conf. Antennas and Propag. (EuCAP), April 2016, pp. 1-5.

[8] P. B. Papazian, C. Gentile, K. A. Remley, J. Senic, and N. Golmie, "A Radio Channel Sounder for Mobile Millimeter-Wave Communications: System Implementation and Measurement Assessment," IEEE Trans. Microw. Theory Techn., vol. 64, no. 9, pp. 2924-2932, Sep. 2016.

[9] R. Zetik, M. Kmec, J. Sachs, and R. S. Thomä, "Real-Time MIMO Channel Sounder for Emulation of Distributed Ultrawideband Systems,' Int. J. Antennas and Propag., vol. 2014, p. 16, 2014.

[10] F. Bao, L. Arend, S. Bertl, and J. Detlefsen, "Application of FMCW Radar Principle for fast Inhomogeneity Identification on Transmission Lines," in 6th German Microw. Conf. (GeMiC), March 2011, pp. 1-4.

[11] T. Poguntke and K. Ochs, "Linear Time-Variant System Identification using FMCW Radar Systems," in IEEE 59th Int. Midwest Symp. Circuits Syst. (MWSCAS), Oct 2016, pp. 1-4.

[12] C. E. Cook and M. Bernfeld, Radar Signals: An Introduction to Theory and Application, ser. Artech House Radar Library. Artech House, 1993.

[13] M. A. Richards, Fundamentals of Radar Signal Processing, 2nd ed. Madison: McGraw Hill Professional, 2013.

[14] T. Hauschild and R. Knochel, "Calibration of Short Range FMCWRadars with Network Analyzer Calibration Techniques," in IEEE MTT-S Int. Microw. Symp. Digest, vol. 2, June 1998, pp. 969-972.

[15] S. O. Piper, "Receiver Frequency Resolution for Range Resolution in Homodyne FMCW Radar," in Proc. Nat. Telesyst. Conf., 6 1993, pp. 169-173.

[16] M. Abramowitz and I. Stegun, Handbook of Mathematical Functions: With Formulas, Graphs, and Mathematical Tables, ser. Applied mathematics series. Dover Publications, 1972, vol. 9.

[17] J. L. López and P. J. Pagola, "A Simplification of the Stationary Phase Method: Application to the Anger and Weber Functions," Electron. Trans. Numer. Anal. (ETNA), vol. 46, pp. 148-161, 2017. 